\title{
Employing the Mathcad program within the course of Theoretical Mechanics
}

\author{
Alexander V. Kurilin ${ }^{1, *}$ \\ ${ }^{1}$ Moscow Technological Institute, 199334, Moscow, Russia
}

\begin{abstract}
The author shares his experience in using the «Mathcad» software for conducting classes on «Theoretical Mechanics» in the University course. The program «Mathcad» combines a simple user interface and powerful mathematical tools which can be employed for different mechanical problems. It gives invaluable help in evaluating analytically and numerically many difficult integrals, in solving differential equations, in picturing graphics and can also simulate different mechanical phenomena with the computer. This allows significantly to expand the range of topics that can be considered in the standard University course of «Theoretical Mechanics» and gives students an opportunity to concentrate on practical problems, avoiding unnecessary routine of mathematical calculations. As an example the author considers one famous problem of the Lagrange analytical mechanics associated with the body motion in the field of gravity in the presence of stationary holonomic constraints.
\end{abstract}

\section{Introduction}

The University course «Theoretical Mechanics» includes a lot of sections. Some of them, such as kinematics, dynamics, statics, the conservation laws of energy and momentum are already being discussed in the framework of the «General Physics», so their study does not cause great difficulties for students. However, quite a few problems of «Theoretical Mechanics» are associated with the Lagrange analytical mechanics, Hamilton mechanics, variational principles, which use a fairly complex mathematical apparatus and the theory of differential equations. Lacking of comprehensive interdisciplinary connections leads to the fact that most of the students are very hard to perceive these topics. Due to the complexity of the mathematical language insurmountable obstacles sometimes arise for them. And this is not due to the fact that students are poorly taught in higher mathematics and in the theory of differential equations. Indeed many practical problems often do not have analytical solutions and can not be integrated in the class of elementary functions. At the same time modern computer technologies greatly expand our capabilities in problem solving and allow us to investigate (at least numerically) many tasks which are of great practical use. The author has a long practice with the «Mathcad» program employing it for the educational purposes [1]. This computer software [2] allows one to solve numerically any differential equations and it also gives us an opportunity to demonstrate the results of calculations by means of computer animation.

\section{Analysis and discussion}

This paper presents an example of using the «Mathcad» in the course of «Theoretical Mechanics». We consider the problem of a body motion in the field of gravity which is placed on a given smooth curve. This task is a natural generalization of the well-known Brachistochrone problem [3,4] of Johann Bernoulli which was formulated in 1696 . The problem of quickest descent, or the Brachistochrone curve involved the greatest scientists for its solution. We can mention such names as the Gottfried Wilhelm von Leibniz, Jacob Bernoulli, Guillaume François de L'Hôpital, and Isaac Newton. Johann Bernoulli himself has also offered the solution to this problem. Recall its essence. Suppose, that there are two points A and B, which lie in a vertical plane and which are separated by a certain vertical distance $H$ and the distance $L$ horizontally. When a ball rolls from $\mathrm{A}$ to $\mathrm{B}$, which curve yields the shortest duration? Five different solutions to this problem were obtained which concluded that brachistochrone, the steepest descent curve, is a cycloid given by the following parametric equations (see Fig. 1.):

$$
\left\{\begin{array}{l}
x(u)=C \cdot(u-\sin u) \\
y(u)=H-C \cdot(1-\cos u)
\end{array}\right.
$$

A cycloid may be expressed as the trajectory of a point fixed on the circumference of a wheel with radius $R$, when it rolls along a straight line. (see Fig. 2). 


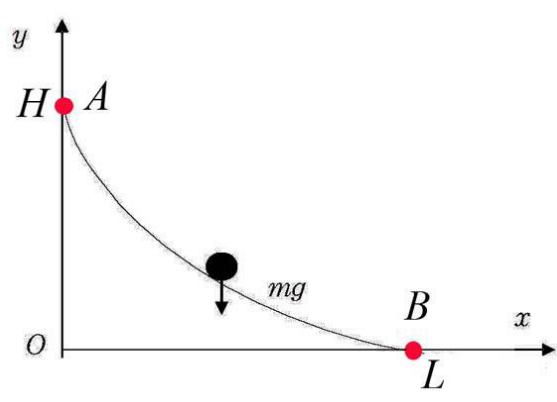

Fig. 1. The Cartesian coordinates of Brachistochrone.

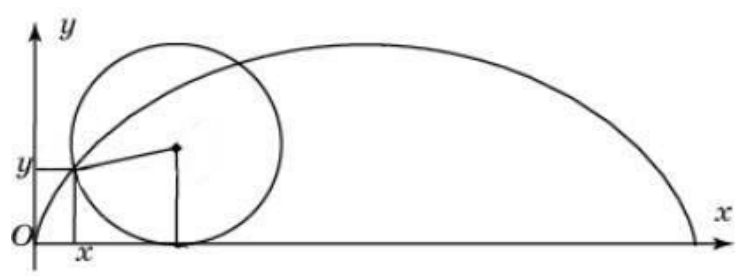

Fig. 2. A point on the rim of a wheel moves along a cycloid.

The $C$ parameter in brachistochrone equation (1) actually coinciding with the wheel radius $R(C=R)$ is associated with Cartesian coordinate of the points $A(0 ; H)$ and $B(L ; 0)$ through the following relationships:

$$
\left\{\begin{array}{l}
L=C \cdot\left(u_{0}-\sin u_{0}\right) \\
H=C \cdot\left(1-\cos u_{0}\right)
\end{array}\right.
$$

where $u_{0}$ is the limit value of the $u$-parameter included in the cycloid equation (1): $0 \leq u \leq u_{0}$.

A direct generalization of the brachistochrone problem is to study the motion of a material point on an arbitrary smooth curve defined by some equation $y=\phi(x)$. It would be interesting to know exactly how much greater is a transit time for a point on a parabola for example in comparison, with the one of brachistochrone movement. We would like to see how it happens and to simulate these motions with the computer. All these tasks can be easily solved with the help of Mathcad. From the point of view of «Theoretical Mechanics» the body motion on a smooth curve can be described by the Lagrangian:

$$
L=\frac{m}{2}\left(\dot{x}^{2}+\dot{y}^{2}\right)-m g y,
$$

with some fixed holonomic constraint on the coordinates of the body:

$$
y=\phi(x) .
$$

Since the Lagrangian (3) does not depend explicitly on time, there is a first integral of motion - the total mechanical energy:

$$
E=\frac{m}{2}\left(\dot{x}^{2}+\dot{y}^{2}\right)+m g y \equiv m g H \quad,
$$

which allows to reduce the problem to the solution of first-order differential equation:

$$
\frac{d x(t)}{d t}=\sqrt{2 g \frac{H-\phi(x)}{1+\phi^{\prime 2}(x)}} .
$$

The analytical solution of this equation can be obtained for the case of brachistochrone (1) and for movement on an inclined plane

$$
y=\phi(x)=H\left(1-\frac{x}{L}\right) .
$$

For all other cases, it is extremely difficult to integrate the motion equation (6) analytically. «Mathcad» allows to investigate this task numerically and to find elegant graphic solutions. As an example, in this article we consider a movement along a parabola defined by the following equation

$$
\phi(x)=H\left(1-\frac{a x}{L}-\frac{b x^{2}}{L^{2}}\right),
$$

and compare it with the motion along the brachistochrone curve. The parameters $a$ and $b$ are related by the condition: $a+b=1$. If $b=0$ we get the motion along a straight line. The time of movement along the parabola (7) can be found using the integral:

$$
T_{1}=\frac{1}{\sqrt{2 g}} \int_{0}^{L} \sqrt{\frac{1+\phi^{\prime 2}(x)}{H-\phi(x)}} .
$$

A complete solution of this problem in «Mathcad» is published in the Internet on the website of the author [5]. Here we point out the major results only.

For numerical calculations the following values of $H=5 \mathrm{~m}$ and $L=4 \mathrm{~m}$ were taken to study the body motion along the curves (1) and (7). The parameters of brachistochrone, which can be obtained from (2) are equal to $\mathrm{C}=3,405 \mathrm{~m}, u_{0}=2,058$. The times of descend along the brachistochrone $T_{0}$ and parabola (if $a=b=$ $0,5) T_{1}(8)$ are given by:

$$
\begin{aligned}
& T_{0}=u_{0} \sqrt{C / g}=1.213 \mathrm{c}, \\
& T_{1}=1,447 \mathrm{c} .
\end{aligned}
$$

The graphs listed below (Fig 3 and Fig 4) show the dependence of Cartesian coordinates of moving bodies on the brachistochrone and on the parabola as functions of time. The blue line corresponds to the movement along the parabola and the red one illustrates the sliding on the brachistochrone. The data of calculations with «Mathcad» are used to simulate the motions along the given curves in real or slow time. The animated video for this task is presented in YouTube [6]. This video was created in «Mathcad» with the command Tools $\rightarrow$ Animation from the main menu of the program. Employing «Mathcad» we solve the differential equation (6) and find the location coordinates of bodies at different times. These data are plotted in $X Y$-plane at various moments to create a stream video that can be viewed by any video player (for details see e.g. [7]). 


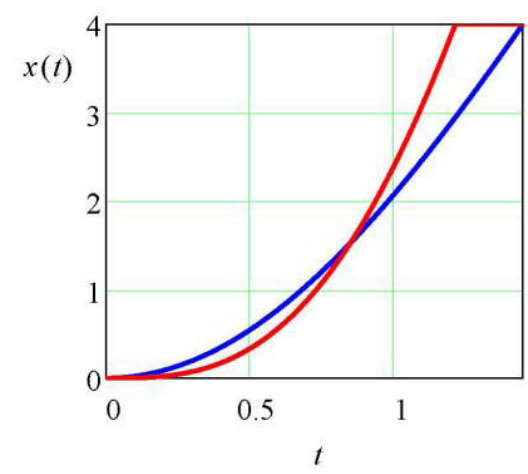

Fig. 3. Horizontal movement along brachistochrone and parabola.

Curiously, that in the beginning the point on the parabola goes ahead the one on the brachistochrone up to 0.85 seconds. However, in the last section of path the point on brachistochrone moves forward and comes to the finish line first.

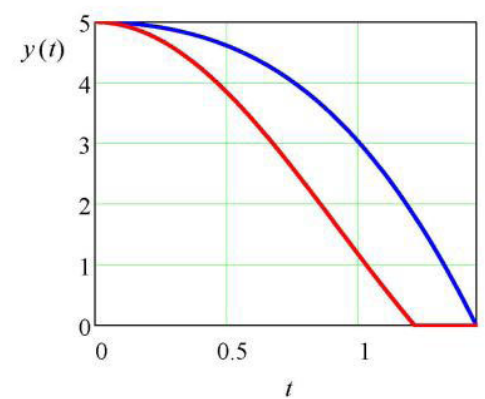

Fig. 4. Vertical movement along brachistochrone and parabola.

\section{Conclusions}

The algorithm developed in this paper can be applied to study the motions along any other smooth curve. It can be used for the educational process both as illustrative material and in the form of individual assignments for students within the course «Theoretical Mechanics» [8].

\section{References}

1. A.V. Kurilin, The use of the Mathcad program in teaching students of technical specialties. In proceedings of the IX International Scientific and Practical conference «Educational Environment. Today and Tomorrow», 57-59 (2014) (in Russian).

2. PTC Mathcad - http://ru.ptc.com/product/mathcad

3. J.J.O'Connor, E.F. Robertson, The brachistochrone problem, http://www-history.mcs.standrews.ac.uk/HistTopics/Brachistochrone.html

4. V. M. Tikhomirov, Kvant, 56 (1986) (in Russian).

5. http://mgopu-math.narod.ru

6. https://youtu.be/ha9OOO_sgLA

7. A.V. Kurilin, Virtual Physical Experiments and Modeling Mechanical Phenomena with «Mathcad». In proceedings of the X International Scientific and Practical conference «Educational Environment. Today and Tomorrow», 382-385 (2015) (in Russian).

8. http://mgopumath.narod.ru/Pages/Brachistochrone.htm 